\title{
Conceptos claves para miembros de la Asociación Europea de ConserVATORIOS INVESTIGACIÓN ARTISTICA \\ Libro blanco del Consejo de la AEC, 2015*
}

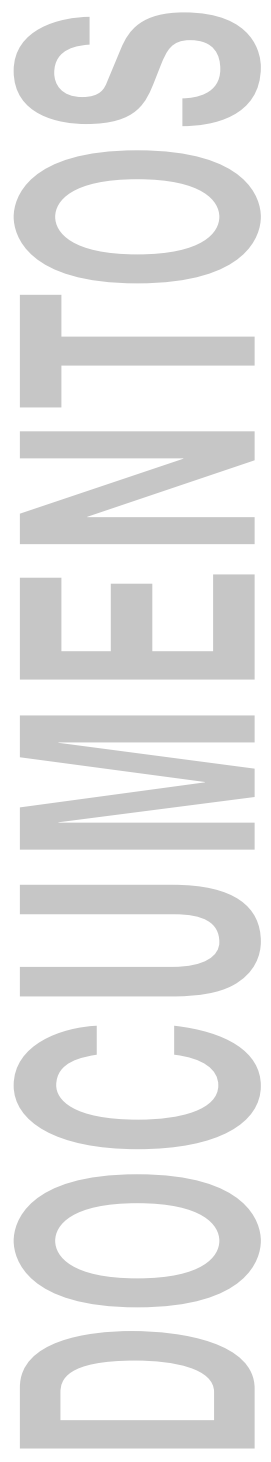

\author{
PRIMERA PARTE: El concepto básico
}

\section{Fundamento}

En opinión de la Asociación Europea de Conservatorios (AEC), la investigación tiene un importante papel que desempeñar en la vida y en la actividad de los conservatorios, como medio para promocionar el conocimiento y el desarrollo de las artes musicales. Del mismo modo, la AEC reconoce que no todos los conservatorios desearán necesariamente participar en actividades de investigación explícitas y que no todos los que sí lo deseen querrán añadirle el título de «investigación artística» a lo que hagan. Asimismo, admite que no solo resulta difícil alcanzar definiciones precisas, sino que también estas podrían restringir las legítimas ambiciones de algunas instituciones miembros en materia de investigación.

Al elaborar este documento conceptual, la AEC desea expresamente apoyar la libertad de las instituciones para decidir por sí mismas qué papel debe desempeñar la investigación en sus actividades (si es que consideran que debe desempeñar alguno). No obstante, llegados a este punto, la AEC viene actuando con la constatación de que un creciente número de sus instituciones miembros está empezando a integrar la investigación de una forma u otra como elemento esencial de su misión.

El concepto de investigación artística de la AEC parte del convencimiento de que debe ser concebida de forma inclusiva y no estar vinculada a una determinada ortodoxia. Se considera una ventaja el hecho de que sea polifacética y se valga de cualquier disciplina o método de investigación relevante para su fin.

* Traducción del siguiente texto original: Artistic Research: An AEC Council White Paper' (2015). Texto realizado por la Asociación Europea de Conservatorios (AEC), acceso 20 de noviembre de 2020, https://www.aec-music.eu/publications/white-paper-on-artistic-research-key-conceptsfor-aec-members. Traducción realizada por el Servicio de Traducción de la Universidad de Alcalá. 


\section{Definición}

Habida cuenta de todo lo anterior, la AEC propone la siguiente definición general:

La «investigación artística» puede definirse como una forma de investigación que posee una sólida base arraigada en la práctica artística y que genera nuevo conocimiento y/o ideas y perspectivas en el seno de las artes, contribuyendo a la excelencia y a la innovación.

Normalmente, la investigación artística muestra la totalidad, o la mayoría, de los siguientes rasgos:

- Suele llevarla a cabo un artista-investigador o tiene lugar mediante la colaboración de artistas dentro de un equipo de investigación.

- Fomenta un diálogo crítico en el seno del campo artístico y también con otros campos de conocimiento relevantes, así como entre los ámbitos académico y profesional.

- Viene respaldada por una reflexión crítica del contenido y/o el contexto del tema de investigación.

- Articula métodos y procesos de trabajo y reflexiona sobre estos.

- Comparte conocimientos profesionales relevantes con el conjunto de la comunidad artística y los divulga en la esfera pública con el fin de enriquecer el conocimiento cultural.

\section{SEGUNDA PARTE: Desarrollo del concepto}

\section{Lugar de la investigación artística en el contexto de investigación general}

La investigación es una búsqueda rigurosa y formalizada de conocimiento y saber; la investigación artística debe encajar en este marco general y, al mismo tiempo, hacer valer su carácter específico y distintivo. Con el fin de ubicar la definición anterior de «investigación artística» en el contexto general de los tipos y las tradiciones de investigación, es preciso ampliar y matizar algunas cuestiones:

- Aunque la definición propuesta de «investigación artística» tiene por objeto hacer referencia a la investigación que se suele llevar a cabo en el contexto de la producción artística (p. ej., en los conservatorios), esto no implica que toda la investigación que se lleva a cabo en un conservatorio sea necesariamente de carácter artístico; hay tipos de investigación que pueden adecuarse perfectamente a los recursos y la perspectiva de un conservatorio, pero que no 
tengan como objetivo principal la promoción del desarrollo de las artes musicales (p. ej., estudios sobre los beneficios de la actividad musical para la salud).

- Aunque la investigación artística tiene una aplicación sumamente práctica, no descarta la investigación pura. De hecho, para poder lograr avances, es probable que el campo de la investigación artística respalde un amplio abanico de actividades componentes, algunas de las cuales pueden contar como investigación pura, otras, como aplicada e incluso otras, como investigación traslacional o del desarrollo.

- La investigación artística no debe interpretarse como algo incompatible con formas más tradicionales de investigación. La investigación artística debe aspirar a los mismos estándares de procedimiento que se aplican en todo el espectro de la investigación -replicabilidad (especialmente de los procedimientos), verificabilidad, justificación de las afirmaciones citando pruebas, etc.-. No obstante, especialmente en áreas como la replicabilidad, debe gozar de libertad para alcanzar estos estándares de formas específicas de su propia naturaleza y de la naturaleza individual y subjetiva de la práctica artística.

- La investigación artística comparte con otros tipos de investigación centrados en el estudio de las artes el objetivo de promocionar el conocimiento y, por ende, el desarrollo de la práctica artística; sin embargo, se distingue por hacer hincapié en el papel esencial que desempeña el artista en sus procesos de investigación. La práctica artística es el espacio en el que surgen sus preguntas y también el destino hacia el que se dirigen sus respuestas.

\section{Características de la investigación artística en el contexto de los conservatorios}

De un modo similar, es preciso formular una serie de puntos adicionales con el fin de explicar en mayor detalle las características que es probable que muestre la investigación artística en el contexto de los conservatorios:

- La centralidad de los procesos y productos artísticos

Los procesos y productos artísticos -actuaciones, composiciones, etc.- son fundamentales para los patrones de trabajo de los conservatorios. Por lo tanto, parece lógico y aconsejable que desempeñen el mismo papel fundamental en cualquier actividad de investigación que se realice en conservatorios, aunque el modo de reflejar este carácter debe ser en todo momento algo que decida cada institución por sí misma. La creciente importancia de la tecnología debe considerarse un campo de oportunidades para desarrollar de manera conjunta la práctica artística y la de investigación. 
- Procesos o productos artísticos en relación con otros elementos

Aunque los procesos o productos artísticos son componentes esenciales de la investigación artística y están integrados en esta, debería permitirse cierta flexibilidad con respecto a cómo y cuándo aplicarlos: en una parte o en la totalidad de las fases del proceso de investigación; y por sí solos o junto con otros elementos de investigación.

- La investigación artística como proceso de colaboración

Cabe la posibilidad de que un proyecto de investigación artística lo lleve a cabo una única persona que no solo tome parte en los procesos artísticos objeto de la investigación, sino que también posea las competencias necesarias en materia de investigación. Sin embargo, dado el carácter multidisciplinario de la investigación artística, es de esperar que la mayor parte de la investigación se base en la colaboración. Muy pocos investigadores son por sí solos expertos en todas las áreas pertinentes. En consecuencia, es habitual que la investigación artística corra a cargo de equipos en los que las competencias y los conocimientos estén distribuidos de forma desigual entre sus miembros.

- Alumnado y profesorado

La investigación en conservatorios puede abarcar tanto el trabajo en el que participen estudiantes en el contexto de su currículum de estudios, como el trabajo desarrollado por el equipo docente como parte de su desarrollo profesional. Aunque es probable que los alumnos participen y se involucren en actividades de investigación principalmente en sus estudios de $2 .^{\circ} \mathrm{y}$, en especial, de 3 . $^{\text {er }}$ ciclo, también recibirán una introducción a los principios de la investigación y a la «actitud de investigación» como parte de sus estudios de $1 .{ }^{\text {er }}$ ciclo.

La investigación durante el 2. ${ }^{\circ}$ ciclo puede que beneficie principalmente a cada alumno, mientras que la investigación durante el $3 .^{\text {er }}$ ciclo y más adelante debería ser capaz de incidir en el conjunto de la comunidad.

\section{- Comunicación de los resultados de la investigación}

Los resultados de la investigación artística se proporcionan en diversos formatos y soportes. Todo investigador artístico tiene la obligación ante la comunidad investigadora de explicar tanto el proceso como el resultado de su investigación, de manera que ambos se ajusten a los estándares habituales de inteligibilidad entre pares que se aplican en la investigación más tradicional. No obstante, la persona que investiga goza de libertad para explorar nuevas formas, más estrechamente relacionadas con el componente artístico, a través de las cuales pueda tener lugar esta dilucidación, ya sea de forma parcial o total, siempre y cuando se tenga en cuenta en todo momento la obligación primordial de que exista una comunicación y difusión claras. 
- Difusión adecuada de los resultados de la investigación

Los procesos y resultados de la investigación deben documentarse y difundirse de manera adecuada, para que estos puedan llegar a la comunidad investigadora, a la comunidad artística y al público en general. La difusión no tiene por qué limitarse a la palabra escrita, aunque en la mayoría de los casos otras formas de comunicación se verán complementadas por elementos escritos y complementarán a estos.

No basta con llevar a cabo un trabajo y considerarlo una «comunicación de los resultados de la investigación», sino que la investigación artística, como disciplina, debe fomentar el conocimiento y el respeto de la diferencia existente entre simplemente aplicar los resultados de una investigación en procesos, y productos artísticos, y tratar de comunicarlos directamente a través de dichos procesos y productos.

\section{Características de la investigación artística que la confirman como disciplina plenamente establecida}

La AEC respalda al creciente número de sus instituciones miembros que han adoptado el concepto de investigación artística. Ve con favor los avances que vienen produciéndose con el fin de que se acabe dando la gama completa de condiciones institucionales típicas de cualquier disciplina de investigación asentada, entre ellas:

- Sus propias asociaciones nacionales e internacionales

- Sus propias revistas

- Sus propios discursos (y no un solo discurso)

- Sus propias figuras de referencias, reconocidas como expertas en la materia

- Sus propios congresos periódicos

- Puestos de docencia a jornada completa en conservatorios

- Formación relevante de nivel doctoral y de desarrollo posdoctoral

- Programas específicos de financiación para investigación

- Financiación para alumnos de posgrado 


\section{TERCERA PARTE: Resumen de las opiniones y políticas generales de la AEC sobre la investigación artística}

\section{La AEC cree que:}

- La investigación artística, como medio para promocionar el conocimiento y desarrollo de las artes musicales, tiene potencial para desempeñar un papel importante en la vida y el trabajo de los conservatorios.

- Es preciso alentar a las instituciones que son miembros de la AEC a que participen en actividades de investigación artística o que deseen hacerlo, a la vez que se respeta a las que no deseen seguir el mismo camino.

- En aquellos casos en los que se lleve a cabo investigación artística en conservatorios, esta resulta útil si, en la medida de lo posible, complementa la actividad artística que constituye la actividad principal de estas instituciones. Esto implica buscar oportunidades para examinar preguntas de investigación que guarden relación tanto con el repertorio más interpretado como con músicas poco conocidas o de reciente creación.

\section{Cómo va a apoyar la AEC el desarrollo de la investigación artística}

La AEC tiene la intención de apoyar a aquellas instituciones que, siendo miembros de la misma, participen en actividades de investigación artística o que se estén planteando hacerlo:

- Proporcionando pautas útiles de lo que podría conllevar (p. ej., a través de publicaciones como el cuaderno Researching Conservatoires [La investigación en los conservatorios] de la AEC y el manual sobre investigación en programas de $2 .^{\circ}$ ciclo).

- Ofreciendo plataformas en las que los investigadores puedan presentar su trabajo y hablar de él con sus pares (p. ej., la Plataforma Europea de Investigación Artística en Música, EPARM, por su denominación en inglés).

- Desarrollando recursos web relacionados con la investigación (como la base de datos Polifonia de proyectos de investigación de alumnos y orientadores; una bibliografía en internet «viva»; o quizás una revista en línea o actas publicadas de la EPARM).

- Supervisando y apoyando continuamente el crecimiento de la investigación y de un «enfoque investigador» en los conservatorios, no solo en la actividad doctoral o posdoctoral, sino también en los ciclos anteriores. 
- Alentando a las instituciones que tratan de desarrollar programas de doctorado y participan en actividad investigadora a hacerlo con arreglo a los siguientes principios:

- Desarrollo cuidado y progresivo

- Obtención de una masa crítica de investigadores y recursos como requisito previo para poner en marcha un programa de doctorado

- Adopción de un enfoque orientado hacia el exterior y basado en el establecimiento de contactos e intercambios con otras instituciones que busquen enfoques investigadores similares

- Apoyo a alumnos y empleados que necesiten establecer y mantener contactos con la comunidad investigadora en general en su área de especialización

- Garantizando que el concepto de investigación artística se comprenda de forma adecuada y general, y que la investigación artística no sea algo que se introduzca solamente debido a presiones externas, como la necesidad de aumentar el número de trabajadores con el título de doctor en conservatorios ${ }^{1 *}$ o de obtener una puntuación elevada en procesos de evaluación o criterios de financiación.

$1^{*}$ En este sentido, la AEC está dispuesta a apoyar a instituciones miembros que reciban presiones del ministerio de su país para que, en contra de sus propios deseos, pasen a tener una plantilla compuesta íntegramente por personas con doctorados. Este apoyo no incluye obstaculizar directamente políticas nacionales, pero sí la redacción de cartas formales para explicar la postura europea general. 